\title{
A Randomised Controlled Trial on Analgesic Effect of Repeated Quadratus Lumborum Block Versus Continuous Epidural Analgesia Following Laparoscopic Nephrectomy
}

Dita Aditianingsih ( $\sim$ ditaaditiaa@gmail.com )

Universitas Indonesia https://orcid.org/0000-0001-6201-2400

Pryambodho Pryambodho

Universitas Indonesia

Naufal Anasy

Universitas Indonesia

Aida Rosita Tantri

Universitas Indonesia

Chaidir Arif Mochtar

Universitas Indonesia

\section{Research article}

Keywords: Epidural analgesia; laparoscopic nephrectomy; postoperative analgesia; patient-controlled analgesia; quadratus lumborum block.

Posted Date: May 7th, 2019

DOI: https://doi.org/10.21203/rs.2.9494/v1

License: (c) (i) This work is licensed under a Creative Commons Attribution 4.0 International License.

Read Full License 


\section{Abstract}

Background Epidural analgesia as the pain management for abdominal surgery has unfavorable side effects. The quadratus lumborum block (QLB) has potential as an abdominal truncal block, however, the analgesic efficacy of QLB compared to epidural analgesia is unknown. This prospective randomized controlled study compared the effectiveness of QLB on postoperative opioid requirement and pain intensity with the epidural analgesia technique in transperitoneal laparoscopic nephrectomy. Methods Sixty-two patients undergoing laparoscopic donor nephrectomy were randomised to receive QLB $(n=31)$ or continuous epidural $(n=31)$. The QLB group received bilateral QLB with $0.3-0.4 \mathrm{ml} / \mathrm{kg}$ bupivacaine $0.25 \%$ and the epidural group received bupivacaine $0.25 \% 6 \mathrm{ml} / \mathrm{h}$ for intraoperative analgesia. As postoperative analgesia, the QLB group received repeated bilateral QLB and the epidural group received the decreased dosage of bupivacaine $0.125 \% 6 \mathrm{ml} / \mathrm{h}$ for 24 hours after surgery completion. The primary outcome was cumulative morphine requirement 24 hours postoperatively. Secondary outcomes included postoperative pain scores, sensory block coverage, Bromage score, postoperative nausea and vomiting (PONV), and duration of urethral catheterisation. Hemodynamic parameters were recorded. Result Postoperative cumulative morphine requirement, pain scores, PONV and Bromage score were not significantly different between the QLB and epidural group. The QLB affected T9-L2, continuous epidural block affected T8-L3 dermatomes. Duration of urethral catheterisation was shorter $(p<0.001)$ in the QLB group. The MAP measured at 24 hours after surgery was lower in the epidural group $(p=0.001)$. Conclusion The repeated QLB had similar cumulative morphine requirement and pain intensity, shorter uretheral catheterization duration, and higher MAP, compared with continuous epidural analgesia after transperitoneal laparoscopic nephrectomy. Trial Registration ClinicalTrial.gov NCT03520205 retrospectively registered on May 9th 2018. Keywords: epidural analgesia; laparoscopic nephrectomy; postoperative analgesia; patient-controlled analgesia; quadratus lumborum block.

\section{Background}

Postoperative pain management holds an important role in laparoscopic living donor nephrectomy patient's recovery. The management of postoperative pain remains a major concern because some patients still demonstrated moderate to severe acute pain not different between laparoscopic or open nephrectomy. ${ }^{1,2}$ The analgesic modalities for laparoscopic nephrectomy are epidural analgesia, intravenous patient-controlled analgesia (PCA) opioid, or the combination of both techniques. Epidural analgesia as the gold standard for abdominal surgery, however, has some side effects such as paresthesia, hypotension, hematomas, impaired motoric limbs and urethral catheterisation that could delay recovery. Opioid also has potential side effects such as sedation, pruritus, nausea, vomiting, and respiratory depression. ${ }^{3}$

Blanco and colleagues in 2007 first introduced quadratus lumborum block (QLB) as the posterior abdominal wall block by injecting local anesthetic in the interfascial plane between quadratus lumborum 
and psoas muscle under ultrasound guidance. ${ }^{4}$ The QLB became an alternative to epidural analgesia in reducing postoperative opioid requirements and pain intensity without central neuraxial block side effects in abdominal surgery. ${ }^{4,5}$ However, the efficacy of QLB as perioperative pain management in transperitoneal laparoscopic nephrectomy is unclear. Therefore, this study compared the effectiveness of QLB on decreasing postoperative opioid consumption and pain intensity with epidural analgesia in donor patients following transperitoneal laparoscopic nephrectomy.

\section{Methods}

\section{Patients and study design}

This prospective open-label randomised controlled study adheres to CONSORT guideline. This study was approved by the Ethics and Research Committee of Universitas Indonesia (0211/UN2.F1/ETIK/2018) and was retrospectively registered in ClinicalTrials.gov (NCT03520205) on May $9^{\text {th }} 2018$. Written informed consents were obtained from all adult patients with ASA I-II who underwent laparoscopic living donor nephrectomy in Cipto Mangunkusumo Hospital, DKI Jakarta. The inclusion criteria included age 18-60 year with $\mathrm{BMI}<30 \mathrm{~kg} / \mathrm{m}^{2}$. The exclusion criteria included inability to communicate, history of allergy or contraindication to local anesthetics, contraindication to epidural or QLB (coagulopathy or infections on the injection site). The research protocol was explained to patient who met the inclusion criteria. All patients were educated about QLB and continuous epidural procedures, how to describe the degree of pain with NRS, and how to use the PCA morphine pump when the pain level (NRS) $\geq 4$ after the surgery. After obtaining a written approval, the patients were randomised either into the QLB group (intervention group) or the epidural group (control group). Randomisation was conducted in block sizes of 4 using a computerised randomisation sequence by independent research assistants. Randomisation allocation number for each subject was written on paper and put in a closed envelope. The envelope was opened by the anesthesiologist who was appointed to perform epidural or QLB for this study. An independent pain team collected the data, statisticians and study investigators were blinded throughout the study.

\section{Technique for Epidural, Quadratus Lumborum Block and Pain Management}

Standard monitoring was placed such as electro cardiogram, oxygen saturation, non-invasive blood pressure monitors and cardiometry ICON ${ }^{\mathrm{TM}}$. General anesthesia induction was conducted with administration of fentanyl $2 \mu \mathrm{g} / \mathrm{kg}$ as co-induction, proceeded with propofol $1-2 \mathrm{mg} / \mathrm{kg}$. Endotracheal tube intubation was facilitated with atracurium $0.5 \mathrm{mg} / \mathrm{kg}$. The ventilator was set to volume control with PEEP $5 \mathrm{cmH}_{2} \mathrm{O}$ and $\mathrm{FiO}_{2} 30-50 \%$, breathing frequency was adjusted with $\mathrm{ETCO}_{2}$ target of $35-45 \mathrm{mmHg}$. The anaesthesia was maintained using sevoflurane with $2.5 \%$ MAC, oxygen compressed air ratio of 40:60, atracurium $0.5 \mathrm{mg} / \mathrm{kg} / \mathrm{hour}$, with bispectral index target of $40-60,0.8$ for fraction of inspired sevoflurane, and train of four ratios $£ 25 \%$. All subjects received fentanyl $1 \mu \mathrm{g} / \mathrm{kg}$ i.v. if there is an increase of change in systolic blood pressure or pulse rate $>20 \%$ from the initial value during surgery and 
the total intraoperative fentanyl usage was recorded. Ephedrine was given if there is a decrease in mean arterial pressure (MAP) less than 65 during the study observation.

Patients in the epidural group had an epidural catheter placement procedure in the left lateral decubitus position after intubation under general anesthesia. After ensuring skin asepsis and draping the area with sterile cover, an 18G Tuohy epidural needle was inserted in vertebral interspace T10-11 and catheter was advanced $5 \mathrm{~cm}$ length within the epidural space. ${ }^{6}$ We observed vacuum epidural catheter aspiration and a test dose of $3 \mathrm{ml}$ bupivacaine $0.25 \%$ with adrenaline 1:200,000 without any change in pulse rate or blood pressure to confirm the position of catheter within the epidural space. Then a continuous epidural infusion of bupivacaine $0.25 \% 6 \mathrm{ml} / \mathrm{h}$ was maintained for intraoperative analgesia. After completion of surgery, the continuous epidural dosage was decreased into bupivacaine $0.125 \% 6 \mathrm{ml} / \mathrm{h}$ for 24 hours postoperatively.

All patients in the QLB group received bilateral ultrasound-guided transmuscular QLB (anterior QLB or QLB3) performed by two anaesthetist consultant (DA, P) after induction and intubation under general anaesthesia. Patients were in supine position with the site to be blocked slightly facing upward by pillow underneath it and table tilting. After ensuring skin asepsis of the area, a 2.0-5.5 MHz convex transducer (C5-1E, DC-70, Mindray, Shenzen China) covered with sterile drapes attached to the inferior lumbar (Petit's) triangle that consisted of iliac crest in the inferior region, the latissimus dorsi muscle in the posterior region and external abdominal oblique muscle in the anterior region). The Shamrock sign appeared on the ultrasound, then a $21 \mathrm{G} 100$-mm peripheral block needle (Stimuplex ${ }^{\circledR}$, BBraun, Mesulngen Germany) was inserted in-plane with ultrasound probe passing in anterior to posterior direction through the QL muscle and reached the border between the QL and psoas major muscle. After confirming negative for blood aspiration, $1 \mathrm{ml}$ normal saline was injected to obtain hydrodissection sign for verifying the needle tip, then a volume of $0.3-0.4 \mathrm{ml} / \mathrm{kg}$ bupivacaine $0.25 \%$ with a maximum of $25 \mathrm{ml}$ was injected on each side (Figure 1). After completion of surgery, the bilateral QLB procedures were repeated with the same regimen as described above.

Neostigmine $0.04-0.07 \mathrm{mg} / \mathrm{kg}$ was given to reverse residual neuromuscular block and patient was extubated when had reached train of ratio ratio of $0.9-1.0$. In the recovery room, patient-controlled morphine i.v. was administered using a portable programmed pump (Perfusorò Space PCA Infusion Pump System, BBraun, Germany). The PCA setting was $1 \mathrm{mg}$ bolus, lockout time 10 minutes and maximum dosage $6 \mathrm{mg}$, without basal opioid infusion. All patients were informed how to use the PCA; first at preoperative anaesthesia visit and once again at the recovery room after the surgery. Ondansetron $4 \mathrm{mg}$ i.v. and omeprazole, setuju $40 \mathrm{mg}$ i.v. were administered every 12 hours to prevent postoperative nausea and vomiting (PONV). 


\section{Study parameters and statistical analysis}

The aim of this study was to compare the analgesic efficacy of QLB with continuous epidural analgesia on postoperative morphine requirement and pain scores with numerical rating scale (NRS). MAP, heart rate, and cardiometry cardiac index were recorded at baseline, during surgery, and 24 hours after anaesthesia recovery. The primary outcome was cumulative morphine requirement in 24 hours after surgery. The secondary outcomes were pain scores at rest and movement, and motoric block at time points 2, 6, 12 and 24 hours after anaesthesia recovery. Pain scores were assessed using NRS ( $0=$ no pain; $1-3=$ mild pain, $4-6=$ moderate pain, $7-10=$ severe pain). Motoric block was evaluated using Bromage score (grade 1 = free movement of leg and feet; 2 = just able to flex knees with free movement of the feet; $3=$ unable to flex knees, free movement of feet; $4=$ unable to move the legs or feet). Sensoric block was assessed after anaesthesia recovery using pinprick and cold loss sensation with ice and alcohol. Time to first morphine requirement, urinary catheterisation duration, PONV, and paresthesia that occur within 24 hours postoperatively were recorded. Those outcomes were documented by the acute pain service team and ward nurses.

Sample size calculation was based on primary hypothesis that opioid requirement in 24 hours after surgery. Based on previous study, the mean (SD) cumulative morphine requirement 24 hours after surgery was $4 \mathrm{mg}(\mathrm{SD}=4.723) .{ }^{7}$ From a preliminary study, the mean $(\mathrm{SD})$ cumulative morphine requirement 24 hours after surgery was $4 \mathrm{mg}(S D=6.09)$. The reduction of $20 \%$ in cumulative morphine requirement was considered as clinically relevant. A sample size of 28 patients in each group was determined with a statistical power of 0.8 and type- 1 error of 0.05 . This study recruited 62 patients to allow $10 \%$ dropouts.

All statistical studies were analysed using Statistical Package for the Social Sciences (SPSS) version 20 (IBM Corp, Armonk, NY). Differences between numerical variable were analysed using the unpaired t-test for normal distribution data and Mann-Whitney test for abnormal distribution data. Differences between categorical variable were analysed using Chi square test. Numerical data with normal distribution was displayed in a mean (standard deviation), abnormal distribution was displayed in the median (interquartile range) values, and as percentage for categorical variables. The analysis was statistically significant if the $p$-value was less than 0.05 .

\section{Results}

We enrolled 62 patients who met the inclusion criteria and signed the informed consent to take part in the study from May to August 2018. The subjects were randomly assigned into two groups and received their allocated intervention. None of the subjects were excluded from the study; therefore, all patients were 
followed-up and included in the final analysis (Figure 2). Between the QLB and epidural group, the demographic baseline and perioperative characteristics of the study subjects were similar. Intraoperative fentanyl requirement was not significantly different between the two groups, however, ephedrine requirement was higher in the epidural group compared to QLB group (5.48 \pm 11.20 vs $9.94 \pm 12.14, p=$ 0.026) (Table 1).

Intraoperative MAP, heart rate and cardiac index (Figure 3) were not significantly different between the QLB and epidural group, however, postoperative MAP measured at 24 hours after surgery was significantly lower in the epidural group $72.26(67.69-76.83) \mathrm{mmHg}$ than those in QLB group 83.33 (78.72-87.95) $\mathrm{mmHg}(p=0.001)$ (See Additional file 1).

Figure 4 shows the cumulative morphine requirement at each time point in 24 hours after surgery were comparable between the QLB and epidural group. Time to first morphine initiation using PCA were not significantly different between the QLB and epidural group (246.03 \pm 245.58 vs $251.94 \pm 222.72$ minutes, $p=0.703$ ) (See Additional file 2). There were 3 subjects in the QLB group and 4 subjects in the epidural group did not need additional morphine at all in 24 hours after anaesthesia recovery.

Pain scores at rest and in movement at each time point in 24 hours after anaesthesia recovery were not significantly different between the QLB and epidural group. The lowest pain scores at rest and in movement were at 2 and 6 hours after anaesthesia recovery in both groups. The Bromage score were not significantly different between the groups. Only 1 subject complained paresthesia in epidural groups. The incidence of PONV in the QLB group was not significantly higher than the epidural group. Subjects in the QLB block group had a significantly shorter duration of urinary catheterisation than the epidural group (Table 2).

The mean total dosage of bupivacaine used in the QLB group was significantly lower than the epidural group (Table 1). The bar in Figure 5 shows the spread of sensory block. Patients in the QLB group had loss cold and pinprick sensations from T9 to L2, compared with had loss cold and pinprick sensations from T8 to L3 in the epidural group. Block-related complications such as local anaesthetic systemic toxicity (LAST), bleeding, infection or neurological deficits, and epidural catheter-related problems such as dislodge, blocking, or leakage, were not found in this study.

\section{Discussion}


Alper and colleagues described that patients undergoing laparoscopic or open nephrectomy had nonsignificant differences in postoperative acute pain scores, additional analgesia requirement, and were at equal risk of developing chronic postsurgical pain (CPSP) at 2 and 6 months after surgery. ${ }^{2}$ Clinical trials of QLB are still limited, but evidence has shown the efficacy of QLB in reducing opioid requirement after cesarean section, laparotomy, laparoscopic procedure, and hip surgery. ${ }^{8-10} \operatorname{In} 2017$, Corso and colleagues reported a case of QLB as postoperative pain management after open nephrectomy. ${ }^{11}$ Pain management is important in order to reduce postoperative pain, promotes recovery, and prevent CPSP in living donor patients. Our study was the first randomised controlled trial that compared the analgesic efficacy of bilateral QLB compared with continuous epidural analgesia following transperitoneal laparoscopic living donor nephrectomy.

Blanco and colleagues performed QLB using $0.2 \mathrm{ml} / \mathrm{kg} 0.125 \%$ bupivacaine in which showed a significant reduction in morphine requirement and visual analogue scores for 48 hours after cesarean delivery. The QLB also showed a superior effect of analgesia lasting from 6 to 48 hours compared with TAP block. ${ }^{4}$ In our study, patients receiving pre- and postoperative QLB using $0.3-0.4 \mathrm{ml} / \mathrm{kg} 0.25 \%$ bupivacaine showed comparable cumulative morphine requirement compared with patients receiving continuous epidural analgesia using $6 \mathrm{ml} / \mathrm{hr} 0.125 \%$ bupivacaine for 24 hours postoperatively. The intraoperative fentanyl consumption was also not significantly different between the two groups. Pain intensity at rest and in movement were not significantly different between both groups, suggesting the QLB had comparable analgesic effect with continuous epidural analgesia during the 24 hours after anaesthesia recovery. The transmuscular QLB (anterior QLB or QLB3) facilitates spread of local anaesthetic into the thoracic paravertebral space, produces prolonged block from 6 to 48 hours and achieving visceral pain relief similar to epidural block. Although this spread has not been clearly proven, the QLB has the potential to provide both somatic and visceral analgesia. ${ }^{12}$ The local anaesthetic spreads to the thoracolumbar fasciae that extensively innervated $\mathrm{A}$ - and $\mathrm{C}$-fiber nociceptors and mechanoreceptors. Therefore, it has a direct effect to lower these high-density sympathetic fibers activity that explains the longer analgesic effect of the QLB. ${ }^{13}$

In our study, the dermatomal coverage of either QLB or continuous epidural block was narrower compared to the QLB coverage from T7 to T12 in a study by Murouichi and colleagues. ${ }^{9}$ However, the spread of local anesthetic covered the dermatome area where incisions were performed. Therefore, the analgesic effect was sufficient for surgical wound pain relief, in accordance with the field of laparoscopic nephrectomy whose dermatomes T8-L1 and the Pfannenstiel incision whose dermatomes T12-L1. 2,14 We found pain level assessment was challenging. The PCA morphine after surgery was intended to treat surgical pain, but urethral catheter discomfort also became a trigger for patient to use PCA. Pain 
characteristics and levels of anxiety after surgery was varied between individuals also considered affecting morphine requirement especially in early postoperative period. ${ }^{15-18}$

Increasing perioperative opioid requirement increases the incidence of PONV and postoperative urinary retention (POUR). ${ }^{19,20}$ In our study, the incidence of PONV was not significantly different, since there was no significant difference in perioperative opioid requirement between the two groups. Patients receiving QLB showed significantly shorter duration of urethral catheterisation than patients with continuous epidural analgesia. Early removal of the urethral catheter is recommended regarding postoperative early recovery because longer urethral catheterisation can affect mobilisation. ${ }^{21}$ Epidural analgesia acts on lumbar and sacral nerve fibers and blocks the bladder detrusor function. Hayami and colleagues demonstrated that urethral catheter removal before discontinuing epidural analgesia had higher incidences of POUR regardless of the amount of opioid use. ${ }^{22}$ The inconvenience from epidural catheter placement also inhibits patient mobilization, while the QLB is performed by bilateral injections without catheter insertion. ${ }^{4,23}$ Both the QLB and epidural group showed similar absent of lower extremity motor blockade represented by the Bromage score $(0-1)$. Assessment of limb motor strength is important which may be a sign of impending neurological deficits in epidural analgesia. ${ }^{24}$ Paddalwar and colleagues reported that epidural using $0.125 \%$ bupivacaine had shown effective labour analgesia and reduced motor block intensity. ${ }^{25}$ In our study, the QLB using $0.25 \%$ bupivacaine relieved pain in the abdominal area as effective as epidural analgesia without limb motor blockade.

The optimal dosage of local anaesthetic for QLB is not known, and a higher dosage may have improved and prolonged the analgesic effect. Local anaesthetic systemic toxicity (LAST) was a concern as the QLB in our study was the bilateral high-volume blocks. However, the mean total bupivacaine requirement in the QLB group was significantly lower than the epidural group. We did not find any LAST symptoms during study observation, and the total dosage of local anaesthetic in both groups were lower than the recommendation for bupivacaine to not exceed $2.5-3 \mathrm{mg} / \mathrm{kg}$ or $175 \mathrm{mg}$ per injection with maximum dosage $400 \mathrm{mg}$ in 24 hours. ${ }^{26-29}$ Compared with the QLB group, MAP in epidural group patients were lower and required more ephedrine during observation. Epidural analgesia has unfavorable side effects such as hypotension especially in patients at risk of hemodynamic instability. It directly inhibits the sympathetic nervous system and decreases vasomotor tone resulting in vasodilation that corresponds to decreased MAP values in the epidural group. ${ }^{11}$ Zhu and colleagues found the combination of thoracic epidural and general anaesthesia had lower systolic, diastolic and pulse pressure compared with general anaesthesia alone in laparoscopic cholecystectomy. ${ }^{30}$ 
Our study had several limitations. There was a lack of blinding because of the QLB was bilateral injections without catheter inserted and the epidural block had a catheter inserted. However, those are the common approaches of the blocks. The spread of block was confirmed only once after anaesthesia recovery, and the analgesic efficacy of the two blocks was assessed only in 24 hours after anaesthesia recovery following the policy in our institution that retains epidural catheter only for 24 hours after laparoscopic surgery. The study subject was representative for the normal BMI population underwent laparoscopic nephrectomy in our institution, therefore, generalizability is limited in other population such as obese patient or clinical context in which continuous QL block is available and epidural can be performed longer than 24 hours.

\section{Conclusions}

The repeated QLB had similar cumulative morphine requirement and pain intensity, shorter uretheral catheterization duration, and higher MAP, compared with continuous epidural analgesia after transperitoneal laparoscopic nephrectomy. The QLB has potential as alternative pain management following laparoscopic nephrectomy. Further studies of the QLB regarding the optimal dosage of local anaesthetic is needed.

\section{Abbreviations}

ASA: American Society of Anesthesiologists classification; BMl: body mass index; CPSP: chronic postsurgical pain; ES: erector spinae; HR: heart rate; L2, L3, L4: second, third, fourth lumbar vertebral body; LAST: local anaesthetic systemic toxicity; MAP: mean arterial pressure; NRS: numerical rating scale; PCA: patient-controlled analgesia; PM: psoas major; PONV: postoperative nausea and vomiting; POUR:

postoperative urinary retention; QLB: quadratus lumborum block; SBP: systolic blood pressure; T7, T8, T9, T12: seventh, eighth, ninth, twelve thoracic vertebral body; TAP: transversus abdominis plane.

\section{Declarations}

\section{Ethics approval and consent to participate}

The study protocol was approved by the Ethics and Research Committee of Universitas Indonesia (0211/UN2.F1/ETIK/2018; protocol no: 18-03-0260; approval date: March 12 ${ }^{\text {th }}$ 2018) and was retrospectively registered on May $9^{\text {th }} 2018$ in ClinicalTrial.gov (NCT03520205). Written consent was obtained from each participant.

\section{Consent to publish}

Not applicable. 


\section{Availability of data and material}

The datasets used and/or analysed in the current study are available from the corresponding author upon reasonable request.

\section{Competing interests}

Nothing to declare

\section{Funding}

The authors would like to thank Universitas Indonesia for the financial supports under PITTA UI grant (2043/UN2.R3.1/HKP.05.00/2018). The financial support aids in data collection and writing the manuscript.

\section{Authors' contributions}

Study design: DA, P;

Performed the blocks: DA, P;

Interpretation of the results: DA, P, AT, CAM;

Writing the manuscript: DA, NA

All authors have read and approved the final manuscript.

\section{Acknowledgements}

Nothing to declare

\section{References}

1. Wisbono E, Wahyudi I, Mochtar CA, Hamid ARA, Sukmono RB. Laparoscopic living donor nephrectomy in cipto mangunkusumo hospital: Postoperative pain and recovery analysis. Adv Sci Lett. 2018; 24: 6704-8. http://dx.doi.org/10.32421/juri.v21i2.28 
2. Alper I, Yuksel E. Comparison of acute and chronic pain after open nephrectomy versus laparoscopic nephrectomy: A prospective clinical trial. Medicine. 2016; 95: e3433(1-4). doi: 10.1097/MD.0000000000003433

3. Sahajananda H, Jyothi, Punithavaty. Renal transplantation with and without epidural analgesia. Indian J Anesth. 2006; 50: 187-92.

4. Blanco R, Ansari T, Riad W, Shetty N. Quadratus lumborum block versus transversus abdominis plane block for postoperative pain after cesarean delivery: A randomized controlled trial. Reg Anesth Pain Med. 2016; 41: 757-62. DOI: 10.1097/AAP.0000000000000495

5. Ueshima $\mathrm{H}$, Otake $\mathrm{H}$, Lin J. Ultrasound-guided quadratus lumborum block: An updated review of anatomy and techniques. BioMed Research International. 2017: 7. https://doi.org/10.1155/2017/2752876

6. Afsham G, Chohan U, Khan A, Chaudry N, Khan ZE, Khan AA. Appropriate length of epidural catheter in the epidural space for postoperative analgesia: Evaluation by epidurography. Anaesthesia. 2011; 66: 913-18. doi: 10.1111/j.1365-2044.2011.06820.x.

7. Abdelsalam K, Mohamdin OW. Ultrasound-guided rectus sheath and transversus abdominis plane blocks for perioperative analgesia in upper abdominal surgery: A randomized controlled study. Saudi J Anaesth. 2016; 10: 25-8. doi:4103/1658-354X.169470

8. Ishio J, Komasawa N, Kido H, Minami T. Evaluation of ultrasound-guided posterior quadratus lumborum block for postoperative analgesia after laparoscopic gynecologic surgery. Journal of Clinical Anesthesia. 2017; 41: 1-4. doi: 10.1016/j.jclinane.2017.05.015.

9. Murouchi T, Iwasaki S, Yamakage M. Quadratus lumborum block: Analgesic effects and chronological ropivacaine concentrations after laparoscopic surgery. Regional Anesthesia and Pain Medicine. 2016; 41: 146-50. doi: 10.1097/AAP.0000000000000349

10. McCrum CL, Ben-David B, Shin JJ, Wright VJ. Quadratus lumborum block provides improve immediate postoperative analgesia and decreased opioid use compared with a multimodal pain regimen following hip arthroscopy. Journal of Hip Preservation Surgery. 2018; 5: 233-9. doi: $10.1093 /$ jhps/hny024

11. Corso RM., Pirracini E., Sorbello M., Bellantonio D., Tedesco M. Ultrasound-guided transmuscular quadratus lumborum block for perioperative analgesia in open nephrectomy. Minerva Anestesiologica. 2017: 1-7. doi: 10.23736/S0375-9393.17.12167-X.

12. Onwochei DN, Børglum J, Pawa A. Abdominal wall blocks for intra-abdominal surgery. BJA Education. 2018; 18: 1-6. DOI: https://doi.org/10.1016/j.bjae.2018.07.002

13. Elsharkawy H, El-Boghdadly K, Barrington M. Quadratus lumborum block: Anatomical concepts, mechanism, and techniques. Anesthesiology. 2019; 130: 322-35.

doi:10.1097/ALN.0000000000002524

14. Wong C. Spinal and epidural anesthesia: Complications and side effect of central neuraxial techniques. Anesthesiology. 2007; 107: 518-9. doi:10.1097/01.anes.0000278887.42847.ab 
15. Wandner LD, Scipio CD, Hirsh AT, Torres CA, Robinson ME. The perception of pain in others: How gender, race and age influence pain expectation. J Pain. 2012; 13: 220-7. doi:

10.1016/j.jpain.2011.10.014.

16. Fillingim RB, King CD, Dasillva MC, Wlliams BR, Rilley JL. Sex gender and pain: A review of recent clinical and experimental findings. J Pain. 2009; 10: 447-85. doi: 10.1016/j.jpain.2008.12.001.

17. Aubrun F, Mazoit JX, Riou B. Postoperative intravenous morphine titration. Br J Anaesth. 2012; 108 : 193-201. doi: 10.1093/bja/aer458.

18. Mortofsky DI. Experimental pain. In: Mortofsky DI, Lomranz J, editors. Pain and Aging. United States: Springer, 1997; 77-80.

19. Odom FJ, Rayens MK, Gokun Y, Jalota L, Radke O, Hooper V, et al. The relationship of pain and nausea in postoperative patients for one week after ambulatory surgery. Clin J Pain. 2015; 31: 84551. doi: 10.1097/AJP.0000000000000170.

20. Jenkins LC, Lahay D. Central mechanisms of vomiting related to catecholamine response: Anaesthetic implications. Can Anaesth Soc J. 1971; 18: 434-41.

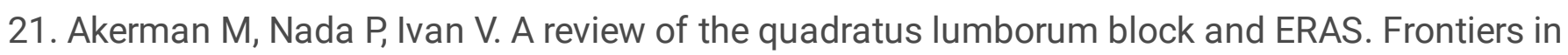
Medicine. 2018; 5: 1-7. doi: 10.3389/fmed.2018.00044

22. Hayami S, Ueno M, Kawai M, Kuriyama T, Kawamata T, Yamaue H. Optimal timing of removal of epidural and urethral catheters to avoid postoperative urinary retention undergoing abdominal surgery. Dirg Surg. 2018; 1-5. doi: 10.1159/000490199.

23. Thurm M. Kroeger Dahlin BI, Winso O, Ljungberg B. Spinal analgesia improves surgical outcome after open nephrectomy for renal cell carcinoma: A randomized controlled study. Scandinavian Journal of Urology. 2017; 5: 277-81. doi: 10.1080/21681805.2017.1318300

24. Shafiq F, Mohammad H, Khalid S. Complications and interventions associated with epidural analgesia for postoperative pain relief in a tertiary care hospital. MEJ Anesth. 2010; 20: 827-32.

25. Paddalwar S, Nagrale M, Chandak A, Shrivastava D, Papalkar J. A randomized, double-blind, controlled study comparing bupivacaine $0.125 \%$ and ropivacaine $0.125 \%$, both with fentanyl $2 \mu \mathrm{g} / \mathrm{ml}$, for labor epidural analgesia. Indian Journal of Pain. 2013; 27: 147-53. doi:4103/0970-5333.124599

26. University of lowa Health Care. Maximum recommended doses and duration of local anesthetics. 2018. https://medicine.uiowa.edu/iowaprotocols/maximum-recommended-doses-and-duration-localanesthetics. Accessed 1 April 2018.

27. Berde CB, Strichartz GR. Local anesthetics. In: Miller RD, Eriksson LI, Fleisher LA, Wiener-Kronish JP, Young WL, editors. Miller's Anesthesia. Amsterdam: Elsevier Academic Press, 2015; 1028-

28. McGee, DL. Local and topical anesthesia. In: Roberts JR, Custalow CB, Thomsen TW, editors. Roberts and Hedges' Clinical Procedures in Emergency Medicine and Acute Care. Amsterdam: Elsevier Academic Press, 2019; 519-

29. Nolan, JP. Anaesthesia and neuromuscular block. In: Brown MJ, Sharma P, Mir FA, Bennett PN, editors. Clinical Pharmacology. Amsterdam: Elsevier Academic Press, 2019; 308- 
30. Zhu J. Zhang X, Yang H. Effects of combined epidural and general anesthesia on intraoperative hemodynamic responses, postoperative cellular immunity, and prognosis in patients with gallbladder cancer: A randomized controlled trial. Medicine 2017; 96: 1- doi: 10.1097/MD.0000000000006137.

\section{Tables}

Table 1. Patient baseline and perioperative characteristics.

\begin{tabular}{lccc}
\hline \multicolumn{1}{c}{ Variables } & QLB group & Epidural group & $p$ value \\
& $(\mathbf{n}=\mathbf{3 1})$ & $(\mathbf{n = 3 1 )}$ & \\
\hline Sex & & & 1.000 \\
$\quad$ Men (\%) & $10(67.74)$ & $20(64.51)$ & \\
$\quad$ Women (\%) & $38.29 \pm 12.97$ & $39.97 \pm 11.49$ & 0.447 \\
Age (years) & $64.34 \pm 10.10$ & $62.72 \pm 10.06$ & 0.530 \\
Body weight (kg) & $164.06 \pm 7.62$ & $161.80 \pm 8.74$ & 0.277 \\
Body height (cm) & $24.06 \pm 3.82$ & $24.13 \pm 4.06$ & 0.940 \\
BMI (kg/m²) & & & 0.794 \\
ASA & $18(58.06)$ & $20(64.51)$ & \\
$\quad$ ASA 1 (\%) & $13(41.94)$ & $11(35.49)$ & \\
$\quad$ ASA 2 (\%) & $58.87 \pm 66.00$ & $70.97 \pm 82.43$ & 0.442 \\
Intraoperative fentanyl dosage ( $\mu$ ) & & & \\
Intraoperative ephedrine dosage (mg) & $5.48 \pm 11.20$ & $9.94 \pm 12.14$ & 0.026 \\
Duration of surgery (minute) & $285.6 \pm 42.6$ & $292.2 \pm 44.4$ & 0.551 \\
Duration of anaesthesia (minute) & $354.6 \pm 36.6$ & $357.6 \pm 33.6$ & 0.796 \\
Total bupivacaine dosage (mg) & $200.00 \pm 19.00$ & $253.18 \pm 11.11$ & $<0.001$ \\
\hline
\end{tabular}

QLB, quadratus lumborum block; BMI, body mass index; ASA, american society of anesthesiologist. Data are presented in number (percentage) or mean \pm standard deviation.

[Table 2. Postoperative pain scores and side effects of QLB versus continuous epidural analgesia] 


\begin{tabular}{|c|c|c|c|}
\hline Parameter & $\begin{array}{l}\text { QLB group } \\
\qquad(\mathrm{n}=31)\end{array}$ & $\begin{array}{l}\text { Epidural group } \\
\qquad(\mathrm{n}=31)\end{array}$ & $p$-value \\
\hline \multicolumn{4}{|l|}{ NRS at rest after anaesthesia recovery } \\
\hline Immediately & $2.06 \pm 1.76$ & $2.61 \pm 2.06$ & 0.313 \\
\hline At 2 hours & $1.97 \pm 1.40$ & $2.19 \pm 1.74$ & 0.785 \\
\hline At 6 hours & $1.87 \pm 1.28$ & $1.97 \pm 1.27$ & 0.708 \\
\hline At 12 hours & $2.10 \pm 1.32$ & $2.13 \pm 1.17$ & 0.659 \\
\hline At 24 hours & $2.06 \pm 1.18$ & $2.13 \pm 1.31$ & 0.878 \\
\hline \multicolumn{4}{|c|}{ NRS in movement after anaesthesia recovery } \\
\hline Immediately & $3.23 \pm 1.87$ & $3.52 \pm 2.03$ & 0.617 \\
\hline At 2 hours & $3.16 \pm 1.50$ & $3.19 \pm 1.74$ & 0.863 \\
\hline At 6 hours & $3.03 \pm 1.25$ & $3.10 \pm 1.39$ & 0.868 \\
\hline At 12 hours & $3.42 \pm 1.38$ & $3.35 \pm 1.19$ & 0.895 \\
\hline At 24 hours & $3.26 \pm 1.18$ & $3.19 \pm 1.30$ & 0.873 \\
\hline \multicolumn{4}{|l|}{ Bromage score after anaesthesia recovery } \\
\hline Immediately & $1(1-2)$ & $1(1-2)$ & 1.000 \\
\hline At 2 hours & $1(1-2)$ & $1(1-2)$ & 1.000 \\
\hline At 6 hours & $1(1-1)$ & $1(1-1)$ & 1.000 \\
\hline At 12 hours & $1(1-1)$ & $1(1-1)$ & 1.000 \\
\hline At 24 hours & $1(1-1)$ & $1(1-1)$ & 1.000 \\
\hline Parestesia (\%) & $0(0.00)$ & $1(3.22)$ & 0.05 \\
\hline Postoperative nausea and vomitting (\%) & $7(22.58)$ & $5(16.12)$ & 0.07 \\
\hline Duration of urinary catheter (hours) & $37.03 \pm 9.14$ & $42.97 \pm 5.72$ & 0.004 \\
\hline
\end{tabular}

Data are presented as mean \pm standard deviation or median (range) or number (percentage).

\section{Figures}


A
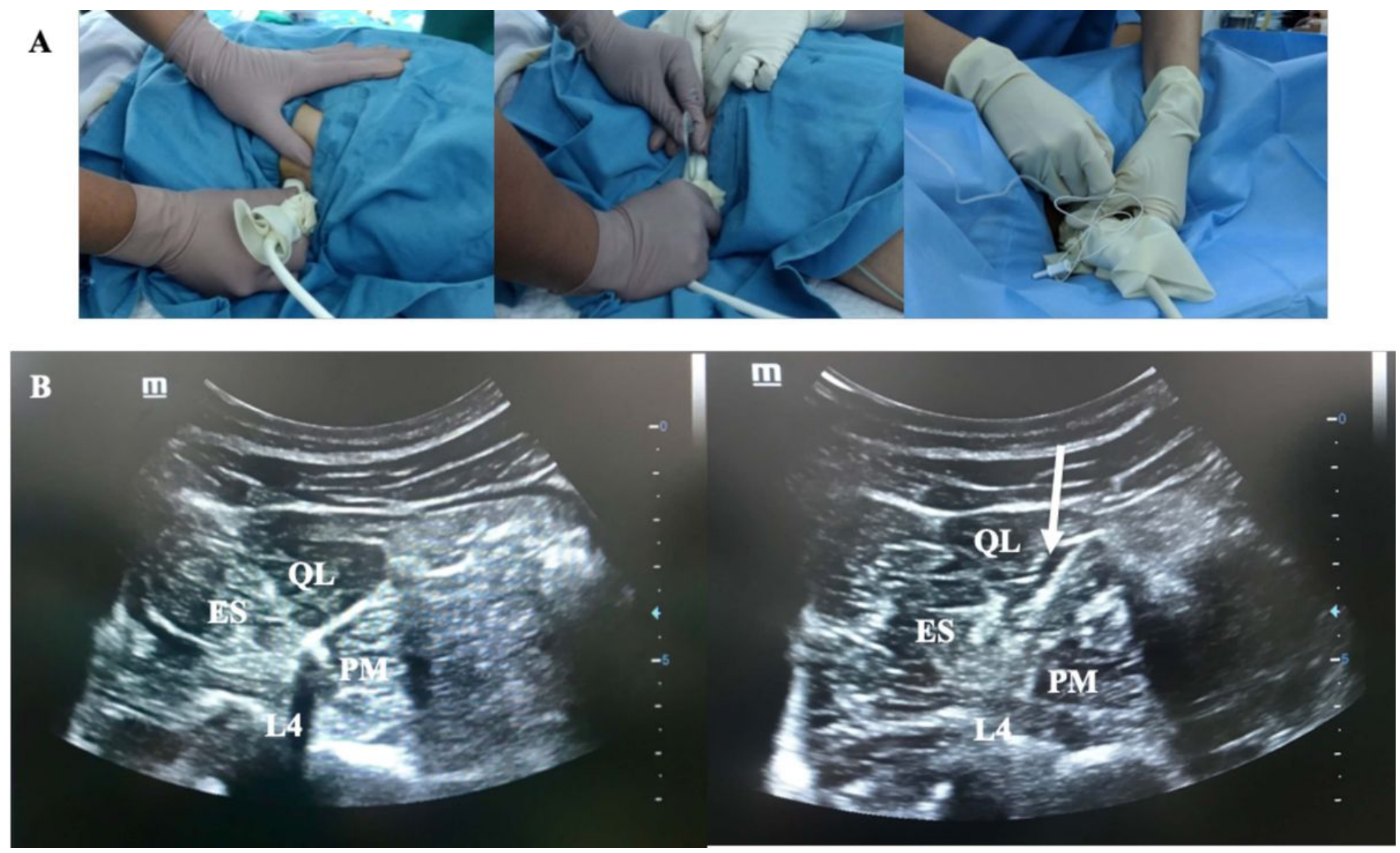

\section{Figure 1}

Anterior quadratus lumborum block sonography A. Patient and ultrasound probe position, a peripheral block needle was inserted in-plane under ultrasound guidance in anteroposterior direction. B. Shamrock sign appeared on the ultrasound, which was the transverse process of the L4 vertebra as a trunk, the posterior ES muscle, the anterior PM muscle, and the lateral QL muscle. B. The needle passed through the quadratus lumborum. White arrow indicates local anaesthetic deposition shown by the widening of hypoechogenic between the QL and PM muscle, and needle trajectory. PM, psoas major; QL, quadratus lumborum; ES, erector spinae; L4, Vertebral body L4. 


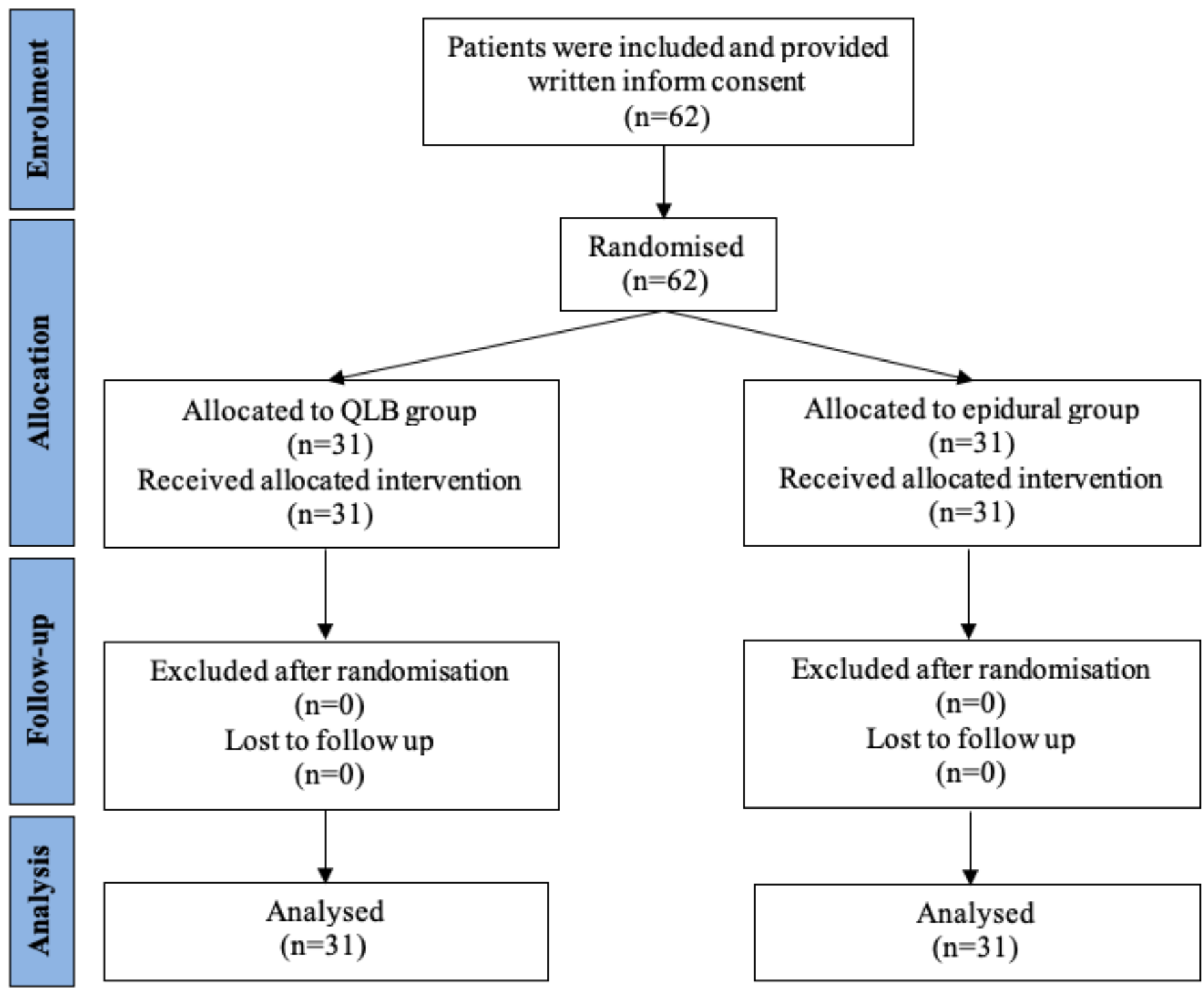

Figure 2

CONSORT flow diagram. 

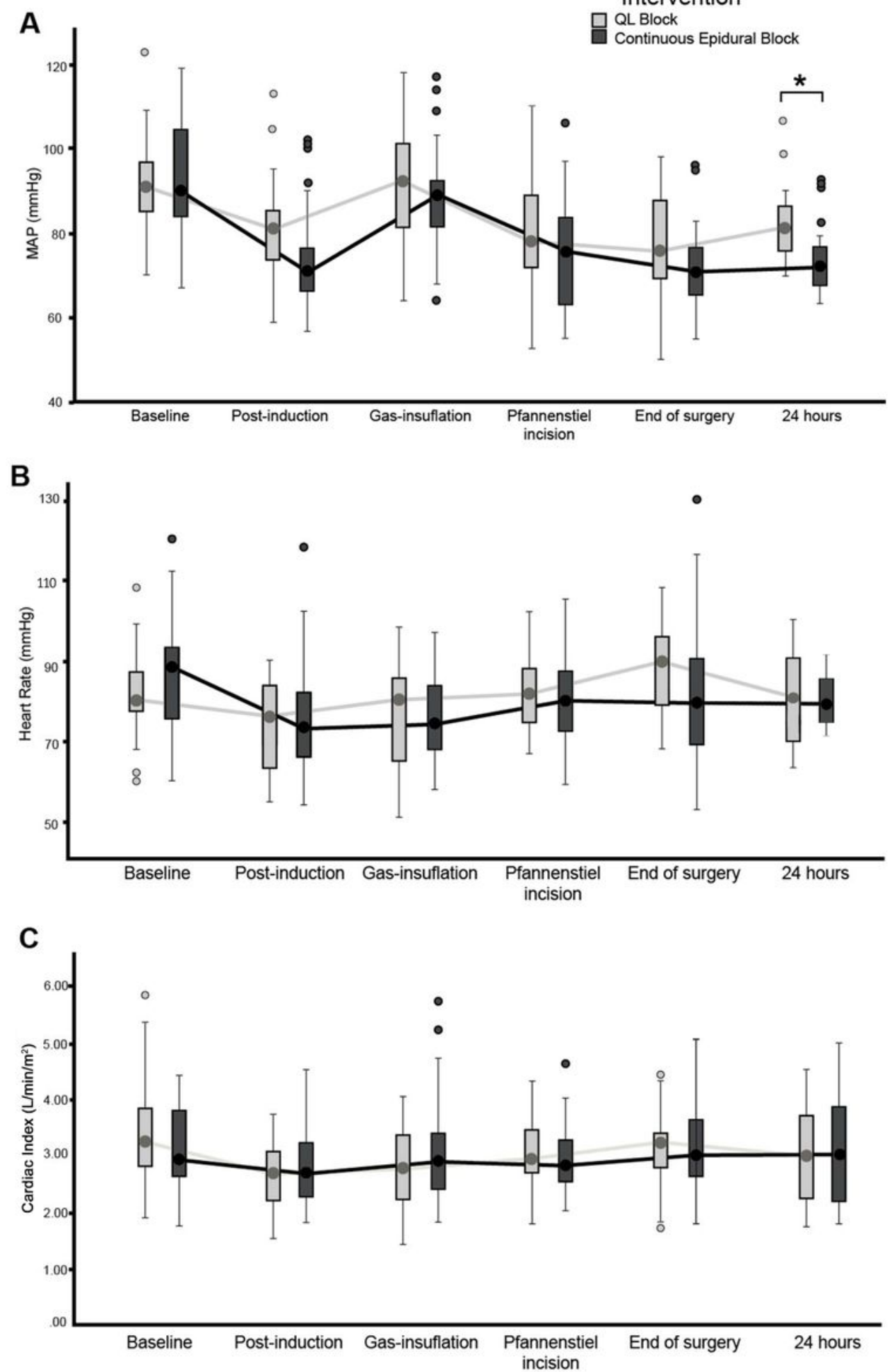

\section{Figure 3}

Intraoperative hemodynamic profile of QLB versus continuous epidural analgesia] The p-values were analysed using Mann-Whitney test, the horizontal lines indicate medians; boxes indicate interquartile range; whiskers indicate range. ${ }^{*} \mathrm{p}<0.05$ is significant. MAP, mean arterial pressure; bpm, beats per minute. 


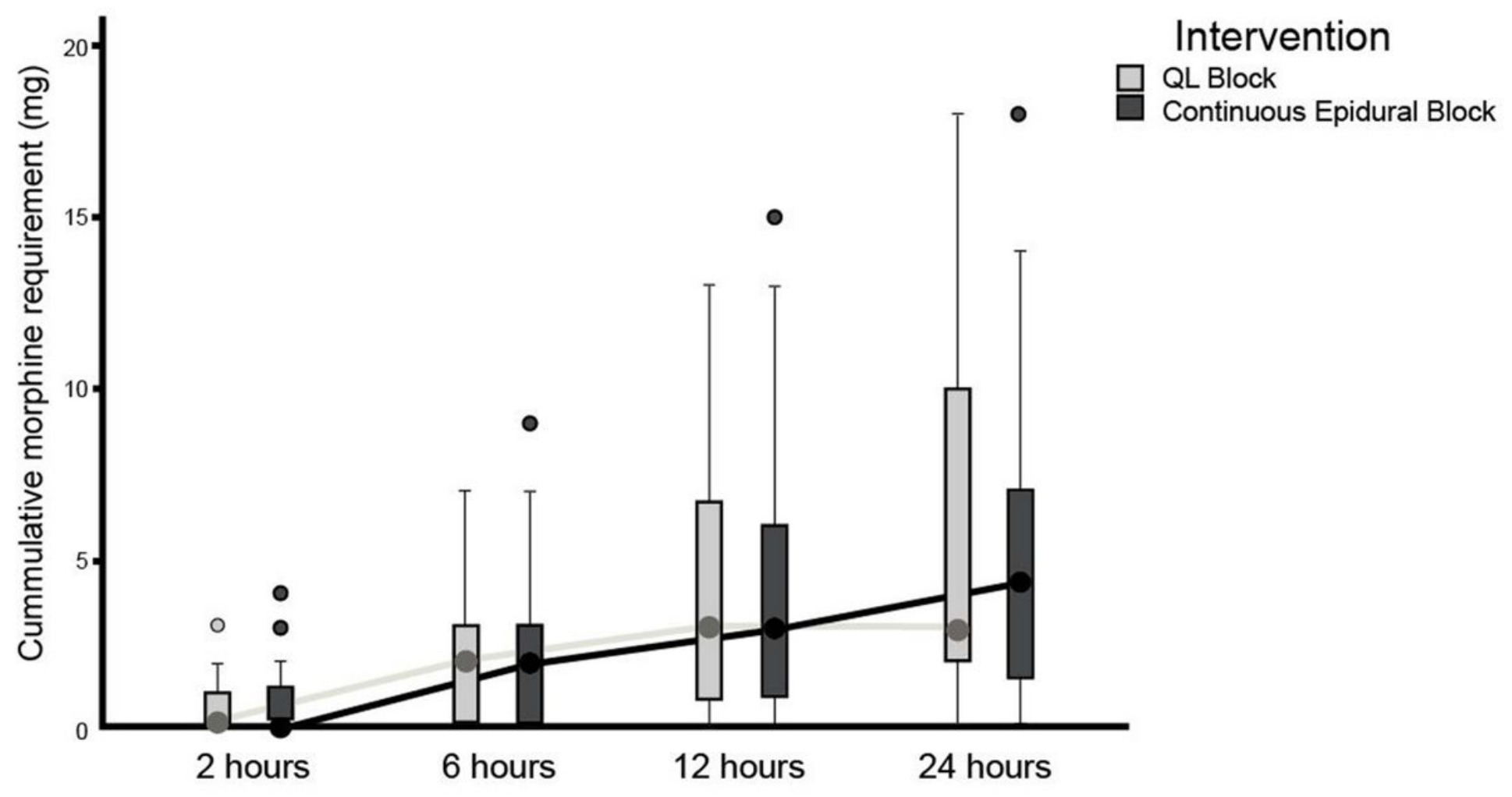

Figure 4

Cumulative morphine requirement of QLB versus continuous epidural analgesia] Median values of cumulative morphine requirement $(\mathrm{mg})$ at each time point after anaesthesia recovery are as follows: 2 hours, $0(0-3)$ to $0(0-4)(p=0.857) ; 6$ hours, $2(0-7)$ to $(2(0-9)(p=0.977) ; 12$ hours, $3(0-13)$ to $3(0-15)(p=0.764) ; 24$ hours, $3(0-18)$ to $4(0-18)(p=0.792)$. The $p$-values were analysed using Mann-Whitney test, the horizontal lines indicate medians; boxes indicate interquartile range; whiskers indicate range. 


\section{QLB \\ $(n=31)$}

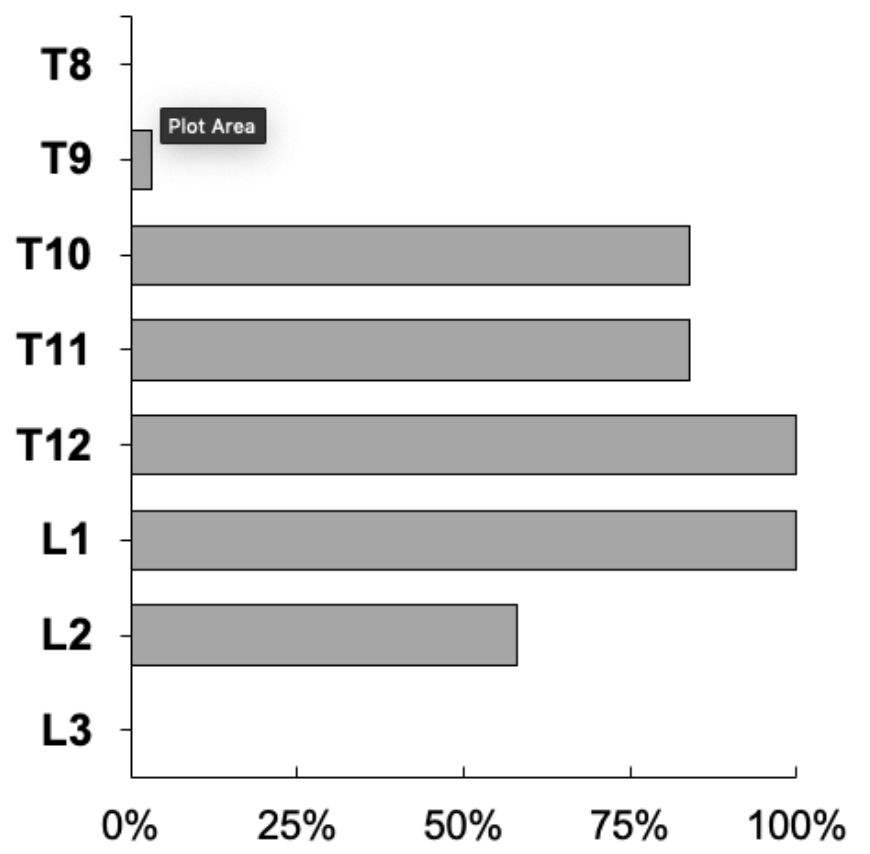

Continuous Epidural

( $n=31$ )

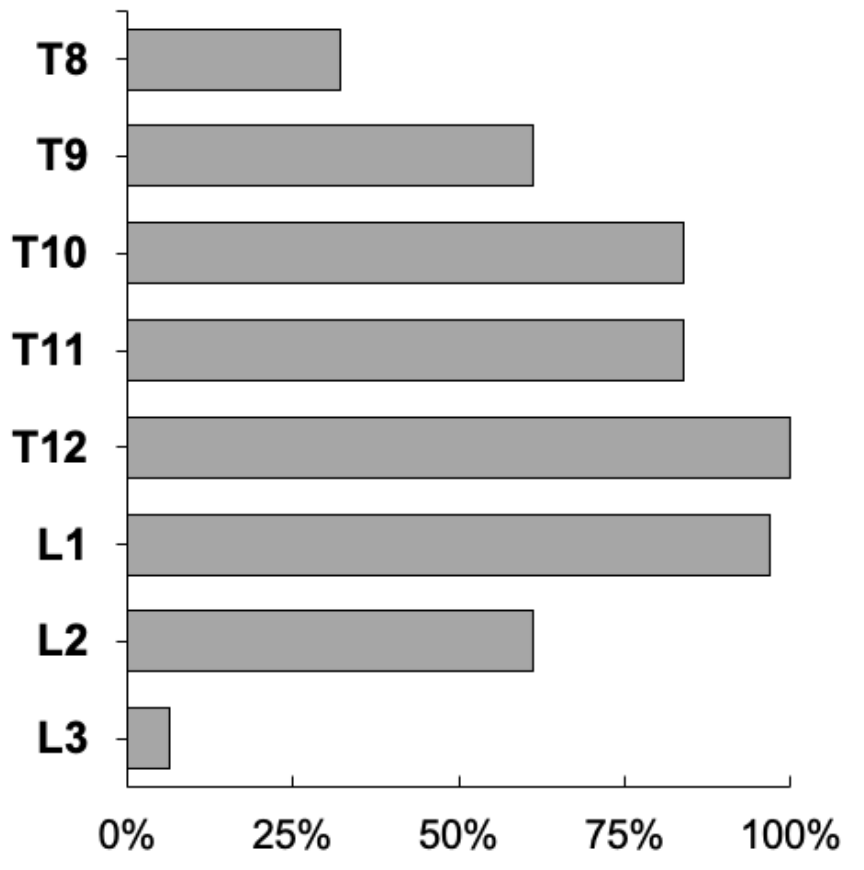

Figure 5

Dermatomal effects of QLB and continuous epidural analgesia] QLB, quadratus lumborum block

\section{Supplementary Files}

This is a list of supplementary files associated with this preprint. Click to download.

- supplement1.docx

- supplement2.docx

- supplement3.pdf 